\title{
Clinician and Health Care Leaders' Experiences with-and Perceptions of-COVID-19 Documentation Reduction Policies and Practices
}

\author{
Amanda J. Moy ${ }^{1}$ Jessica M. Schwartz ${ }^{2}$ Jennifer Withall ${ }^{2}$ Eugene Lucas ${ }^{1,3}$ Kenrick D. Cato ${ }^{2,3,4}$
} S. Trent Rosenbloom ${ }^{5}$ Kevin Johnson ${ }^{5}$ Judy Murphy ${ }^{6}$ Don E. Detmer ${ }^{7}$ Sarah Collins Rossetti ${ }^{1,2}$

\footnotetext{
${ }^{1}$ Department of Biomedical Informatics, Columbia University, New York, New York, United States

${ }^{2}$ Columbia University School of Nursing, New York, New York, United States

${ }^{3}$ NewYork-Presbyterian Hospital, New York, New York, United States

${ }^{4}$ Department of Emergency Medicine, Columbia University Irving

Medical Center, New York, New York, United States

${ }^{5}$ Department of Biomedical Informatics, Vanderbilt University, Nashville, Tennessee, United States

${ }^{6}$ Twin Cities, Minnesota, United States

7 Department of Public Health Sciences, University of Virginia, Charlottesville, Virginia, United States
}

Appl Clin Inform 2021;12:1061-1073.
Address for correspondence Amanda J. Moy, MPhil, MPH, MA, Department of Biomedical Informatics, Columbia University, 622 West 168th Street, PH20, New York, NY 10032, United States (e-mail: am3458@cumc.columbia.edu).

\section{Abstract}

Keywords

- electronic health records

- documentation burden

- physician

- nurse

- survey
Background Substantial strategies to reduce clinical documentation were implemented by health care systems throughout the coronavirus disease-2019 (COVID-19) pandemic at national and local levels. This natural experiment provides an opportunity to study the impact of documentation reduction strategies on documentation burden among clinicians and other health professionals in the United States.

Objectives The aim of this study was to assess clinicians' and other health care leaders' experiences with and perceptions of COVID-19 documentation reduction strategies and identify which implemented strategies should be prioritized and remain permanent post-pandemic.

Methods We conducted a national survey of clinicians and health care leaders to understand COVID-19 documentation reduction strategies implemented during the pandemic using snowball sampling through professional networks, listservs, and social media. We developed and validated a 19-item survey leveraging existing post-COVID19 policy and practice recommendations proposed by Sinsky and Linzer. Participants rated reduction strategies for impact on documentation burden on a scale of 0 to 100 . Free-text responses were thematically analyzed.

Results Of the 351 surveys initiated, 193 (55\%) were complete. Most participants were informaticians and/or clinicians and worked for a health system or in academia. A majority experienced telehealth expansion (81.9\%) during the pandemic, which participants also rated as highly impactful (60.1-61.5) and preferred that it remain received

July 20, 2021

accepted after revision

September 30, 2021
DOI https://doi.org/

10.1055/s-0041-1739518.

ISSN 1869-0327. (c) 2021. The Author(s).

This is an open access article published by Thieme under the terms of the Creative Commons Attribution License, permitting unrestricted use, distribution, and reproduction so long as the original work is properly cited. (https://creativecommons.org/licenses/by/4.0/)

Georg Thieme Verlag KG, Rüdigerstraße 14, 70469 Stuttgart,

Germany 
(90.5\%). Implemented at lower proportions, documenting only pertinent positives to reduce note bloat $(66.1 \pm 28.3)$, changing compliance rules and performance metrics to eliminate those without evidence of net benefit $(65.7 \pm 26.3)$, and electronic health record (EHR) optimization sprints $(64.3 \pm 26.9)$ received the highest impact scores compared with other strategies presented; support for these strategies widely ranged (49.763.7\%).

Conclusion The results of this survey suggest there are many perceived sources of and solutions for documentation burden. Within strategies, we found considerable support for telehealth, documenting pertinent positives, and changing compliance rules. We also found substantial variation in the experience of documentation burden among participants.

\section{Background and Significance}

The widespread adoption and use of electronic health records (EHRs) coupled with a simultaneous increase in regulatory demands have led to a national epidemic of documentation burden among clinicians, including physicians, nurses, and other health professionals. ${ }^{1}$ Physicians in the ambulatory setting spend nearly half their workday in the EHR-the majority of which on administrative and clerical tasks involving clinical documentation.,.$^{2,3}$ Nurses spend approximately one-quarter ${ }^{4}$ to over one-third ${ }^{5,6}$ of their EHR time on documentation. In the acute care setting, nurses document approximately one data point per minute. ${ }^{7}$ There is now scientific consensus that high documentation times and documentation-related stress are associated with clinician burnout, increased medical errors, hospital-acquired infections, and decreased satisfaction. ${ }^{2,8}$ Documentation burden impacts the work-life balance of health care providers and results from an imbalance between EHR usability and satisfaction. EHR design and use factors (e.g., information overload, slow navigation) are significantly associated with high stress and burnout among clinicians. ${ }^{9}$ Poorer perceived EHR usability is also associated with increased burnout among physicians across specialties and practice settings. ${ }^{10}$ Among nurses, low EHR satisfaction has accompanied reports of system-level burden (e.g., usability, interoperability). ${ }^{11}$ Clinical and regulatory demands of entering and consuming EHR data, such as evaluation and management (E\&M) services and meaningful use mandates, 3,12,13 also contribute to documentation burden.

The Quadruple Aim emerged from the Institute for Healthcare Improvement's Triple Aim framework for optimizing health system performance to address clinician burnout on health care outcomes. ${ }^{14}$ Focusing on: (1) enhancing patient experiences, (2) improving population health, and (3) reducing costs, which outline the Triple Aim, the Quadruple Aim also includes: (4) improving health care providers' work-life balance. ${ }^{14}$ Achieving this fourth aim would reconcile the disparity between expectations of patient-centered care and clinician capacities; however, it is contingent on several policy implications, including changes in regulatory and accreditation requirements for and prac- tices around electronic documentation (e.g., reimbursement, quality measures). ${ }^{15}$

During the coronavirus disease-19 (COVID-19) pandemic, health care systems nationwide promptly deployed informatics infrastructure to support clinical and operational pandemic responses (e.g., policies, procedures) at respective institutions. These actions ranged from building EHR-based tools to standardize processes (e.g., data analytics) ${ }^{16}$ and configuring new EHR workflows, ${ }^{17}$ to transitioning to and scaling up telehealth. ${ }^{16,17}$ Various policies impacting clinician documentation burden were enacted, including Centers for Medicare and Medicaid Services (CMS) changes (e.g., telehealth waivers), ${ }^{18}$ removing nonessential administrative activities, ${ }^{19}$ and statebased relaxation of documentation requirements (e.g., recordkeeping for patient treatment and evaluation, and billing). ${ }^{20}$ This natural experiment provides an opportunity to broadly study the impact of billing and regulatory policy "relaxations" on documentation burden, ${ }^{19}$ which would otherwise not be achievable under conventional circumstances. The pandemic brought to the forefront the enduring tension between documentation and direct patient care, and resulted in the reevaluation of existing practices and policies and the revival of prior documentation approaches and processes. ${ }^{19}$ Therefore, investigating COVID-19 documentation reduction strategies is critical to the advancement of sustainable approaches to alleviate documentation-related stress, reduce clinician burnout, and improve patient safety and care quality.

Between January and February 2021, Columbia University, Vanderbilt University, and University of Virginia investigators hosted a National Library of Medicine-funded scientific meeting, convening stakeholders with the goal of reducing clinical electronic documentation burden. Leveraging Sinsky and Linzer's recommendations, ${ }^{19}$ we developed a survey to inform the 25 by 5: Symposium to Reduce Documentation Burden on US Clinicians by $75 \%$ by $2025(25 \times 5)$ and generate knowledge on the impact of COVID-19 documentation reduction strategies on documentation burden.

\section{Objectives}

We conducted a survey to assess the experiences and perceptions associated with COVID-19 documentation reduction strategies and their potential impact on 
documentation burden among clinicians and health care leaders. The overarching goal was to facilitate the prioritization and implementation of effective documentation reduction strategies beyond the pandemic.

\section{Methods}

\section{Study Design and Data Collection}

We conducted an anonymous web-based survey using Qualtrics $^{21}$ (Qualtrics, Provo, Utah) over a 6-week period from mid-November 2020 to January 2021. We recruited clinicians and health care leaders nationally to complete a selfadministered survey through two channels: (1) snowball sampling via email invitations sent to and forwarded by clinicians, health care leaders, and colleagues, including professional listservs (i.e., American Medical Informatics Association [AMIA], American College of Medical Informatics, New England Nursing Informatics Consortium, Alliance for Nursing Informatics), and (2) social media (i.e., Twitter, LinkedIn, Facebook). Invitations to participate included a direct survey hyperlink. We promoted the survey through a panel presentation on documentation burden and networking sessions at the 2020 AMIA Annual Symposium. These data collection techniques broadened outreach and generalizability of data as best as possible, but did not permit calculation of a survey response rate.

\section{Survey}

\section{Survey Development}

We developed a survey leveraging existing post-COVID-19 policy and practice recommendations proposed by Sinsky and Linzer. ${ }^{19}$ The 19-item survey captured information on COVID-19 documentation reduction strategies experienced, which strategies participants preferred to remain permanent, perceptions of the strategies' potential to reduce documentation burden, and two free-text questions on additional documentation reduction strategies not described elsewhere in the survey (see - Supplementary Table S1 [available in the online version only]). Our $25 \times 5$ Steering Committee, comprised of clinicians, informatics experts, and health care leaders, worked jointly in survey development.

\section{Documentation Reduction Strategies}

Eight survey items focused on core COVID-19 documentation reduction strategies implemented (e.g., "verbal orders permitted in hospital setting," "telehealth expansion"). Eleven items focused on additional documentation reduction strategies that may have been instituted at organizations (e.g., "reduced frequency of order re-signatures," "login optimization"). For each core item, participants were asked to indicate whether: (1) they "experienced the strategy," and (2) "prefer [the strategy] to remain permanent" using checkboxes. Lastly, participants were asked to: (3) "rate the projected impact of each strategy on reducing documentation burden" based on a sliding scale from 0 (low impact) to 100 (high impact) in 10-point increments. Identical measures (outlined in 1-3) were collected for each additional item with the exception of "prefer [the strategy] to remain permanent," which was replaced with asking the participant if they would "support implementing the strategy." We added two additional free-text questions to collect information on any additional clinical documentation reduction strategies that participants experienced-related and/or unrelated to COVID-19-which were not originally captured in Sinsky and Linzer's recommendations. ${ }^{19}$

\section{Demographics}

We tailored and incorporated three professional demographic questions based on fields collected by the AMIA ${ }^{22}$ and the American Board of Medical Specialties to suit our needs: (1) profession, (2) specialty, and (3) work setting. Participants had the option of selecting up to three choices for each of the three questions using checkboxes. Location of survey completion was determined through data provided in Qualtrics.

\section{Content Validity}

We elicited feedback on the survey from clinical and informatics experts to determine face and content validity according to Polit and Beck's recommendations. ${ }^{23}$ Steering Committee members identified 16 experts who were contacted directly through email, of whom half responded. Experts were asked to rate the relevance of each strategy for its ability to "assess perceptions of documentation burden reduction strategies" using a scale from 1 (not relevant) to 4 (highly relevant) and to provide overall feedback on face validity using an anonymous web-based Qualtrics ${ }^{21}$ survey. Using the results, we calculated a content validity index on the scale (S-CVI) and item (I-CVI) level. ${ }^{23}$ We determined that the S-CVI was 0.78 , and the I-CVI ranged from 0.33 to 1 . We refined our survey for clarity and incorporated additional items based on their written feedback and comments. For example, we included two additional questions on COVID19-related practice changes that were not described in Sinsky and Linzer ${ }^{19}$; these questions better captured ambulatory-specific practice changes. We also collapsed three questions on telehealth in a single item and used conditional logic that displayed detailed telehealth questions if a participant indicated they had experienced or would endorse telehealth changes. We made this decision given telehealth questions were rated with the lowest relevance by experts (0.33).

\section{Data Analysis}

We conducted descriptive analyses on all completed surveys defined as $\geq 80 \%$ complete. We categorized "profession" into three mutually exclusive role categories: prescribing provider (i.e., physician, advanced practice nurse, physician assistant), registered nurse, and other. Independently, two authors (A. J. M. and J. W.) performed deductive thematic analysis on additional COVID-19 clinical documentation reduction experiences and any additional changes to documentation at any time that participants described in freetext based on the six domains established in the American Nursing Informatics Association (ANIA) conceptual framework on addressing burden: reimbursement, regulatory, 
quality, usability, interoperability/standards, and self-imposed (see - Supplementary Table $\$ 2$ [available in the online version only]). ${ }^{24}$ Two authors (A. J. M. and J. W.) organized and reorganized discordant results until they reached a consensus on the domain(s); the domains identified were reviewed by all co-authors.

\section{Results}

Of the 351 surveys initiated, 193 (55\%) were complete. Among these surveys, most participants reported one profession (42.5\%), while over a quarter reported three professions. The largest proportions of participants were informaticians (40.4\%), registered nurses (36.3\%), and/or physicians (34.7\%) (-Table 1 ); nearly half were prescribing providers. Of the participants who reported multiple professions, informatician-registered nurse (18.9\%) and informatician-physician (7.2\%) were selected most frequently (-Fig. 1). Approximately $48 \%$ of participants worked for a health system, followed by academia (32.6\%) and hospital (32.4\%). The top three specialties selected were internal medicine (26.9\%), family medicine (6.7\%), and pediatrics (6.2\%). -Fig. 2 displays the geographic distribution of the survey responses for which we have the information. We received responses from participants in 37 states, including the District of Columbia, and 10 international responses. Most participants were from Minnesota (11.4\%), New York (9.8\%), California (7.8\%), and Pennsylvania (6.2\%). The following section describes results tallied among completed surveys $(n=193)$.

\section{Quantitative Analysis}

\section{Core COVID-19 Documentation Reduction Strategies}

Experience of Strategies and Preference for Strategies Of the eight COVID-19 documentation reduction strategies identified in the survey ( - Table 2), most respondents experienced "telehealth expansion" (81.9\%), "changed coding for telehealth visits for evaluation and management" (67.9\%), and "disease-specific workflows such as COVID-19 express lanes or order sets" (58.5\%); participants who experienced these strategies equally preferred that they remain postpandemic: $90.5,87$, and $76.1 \%$, respectively (- Table 2). Fewer participants reported experiencing "verbal orders permitted in hospital setting" (29.5\%), "moving laboratory testing to specialized testing centers" (31.1\%), and "waived requirement that nursing staff develop and keep current nursing care plan for each patient" (31.6\%). Similarly, these participants less preferred these strategies remain: 47.4, 36.7, and $45.9 \%$, respectively. Few notable differences existed in the experience of and preference for these strategies between role categories except role-specific strategies (e.g., verbal orders, durable medical equipment requirements, telemedicine coding, nursing care plans) (-Fig. 3). With the exception of "verbal orders permitted in hospital setting" $(37.7 \pm 30)$ and "moving laboratory testing to specialized testing centers" $(42.3 \pm 30.6)$, all COVID-19 reduction strategies (including telehealth-related subquestions) were rated 50 or more out of 100 on burden-reducing impact on average; telehealth achieved the highest average impact ratings (range: 60.161.5) relative to all other core strategies. Prescribing providers consistently rated strategies lower impact on average relative to other roles excluding providing telehealth services from home (-Fig. 3).

\section{Additional Documentation Reduction Strategies}

\section{Experience of Strategies and Support for Strategies}

Fewer than half the participants experienced each additional documentation reduction strategy (-Table 2). Participants reported experiencing "EHR optimization sprints" (43.5\%), "documenting only pertinent positives to reduce note bloat" (40.4\%), and "monitor and improve EHR use measures" (39.4\%) at the highest proportions. Strategies including "reduced frequency of order re-signatures" (11.9\%), "elimination of order requirement for low-risk activities/interventions" (15\%), and "changes to compliance rules and performance metrics to eliminate those without evidence of net benefit" (18.7\%) were least experienced. While participants' experience of additional documentation reduction strategies was low, participants supported the strategies at higher proportions. Among additional strategies, participants supported implementing "EHR optimization sprints" (63.7\%), "eliminate alerts without evidence of net benefit" (60.6\%), and "documenting only pertinent positives to reduce note bloat" (59.1\%) at the highest proportions. These strategies were also rated moderately high for mean impact on burden reduction (range: 66.159.7). Other strategies including "changes to compliance rules and performance metrics to eliminate those without evidence of net benefit" $(65.7 \pm 26.3)$ and "device integration/efficient data capture" $(62.4 \pm 30.5)$ were less supported by participants but rated highly for impact (- Table 1). The least supported strategies were "reduced frequency of order re-signatures" (34.7\%), "increased use of documentation assistance" (42\%), and "elimination of order requirement for low-risk activities/interventions" (44\%). Nevertheless, "increased use of documentation assistance" received moderately high ratings for mean impact $(60.6 \pm 28.1)$. Prescribing providers were more likely to support additional strategies relative to other roles (-Fig.4), but consistently rated additional strategies lower impact on average except for use of documentation assistance, medication reconciliation by support staff, and changes to compliance rules and performance metrics (-Fig. 4).

Additional Experiences Reported in Free-Text Responses Seventy participants (36.3\%) identified additional experiences with clinical documentation reduction during the pandemic through free-text responses ( - Table 2), and one-third reported additional changes to documentation at any time that had increased (64.4\%) or decreased (58.6\%) documentation burden. Among those, one-quarter reported experiencing both increases and decreases from documentationrelated reduction strategies. 
Table 1 Professional demographics among all respondents stratified by survey completion status

\begin{tabular}{|c|c|c|c|}
\hline Demographic variable & $\begin{array}{l}\text { Complete } \\
N(\%)\end{array}$ & $\begin{array}{l}\text { Incomplete } \\
N(\%)\end{array}$ & $\begin{array}{l}\text { Totals } \\
N(\%)\end{array}$ \\
\hline Total $^{\mathrm{a}}$ & $193(73.1)$ & $71(26.9)$ & $264(100)$ \\
\hline \multicolumn{4}{|l|}{ Profession $^{\text {b }}$} \\
\hline Informatician & $78(40.4)$ & $37(52.1)$ & $115(43.6)$ \\
\hline Physician & $67(34.7)$ & $16(22.5)$ & $83(31.4)$ \\
\hline Registered nurse & $70(36.3)$ & $31(43.7)$ & $101(38.3)$ \\
\hline Chief Nursing Informatics Officer/Chief Nursing Officer (CNIO/CNO) & $24(12.4)$ & $5(7)$ & $29(11)$ \\
\hline Researcher & $22(11.4)$ & $6(8.5)$ & $28(10.6)$ \\
\hline Chief Medical Information Officer/Chief Medical Officer (CMIO/CMO) & $19(9.8)$ & $5(7)$ & $24(9.1)$ \\
\hline Advanced practice nurse & $20(10.4)$ & $5(7.9)$ & $25(9.5)$ \\
\hline Educator & $20(10.4)$ & $9(12.7)$ & $29(11)$ \\
\hline Management & $9(4.7)$ & $4(5.6)$ & $13(4.9)$ \\
\hline Health care administrator & $6(3.1)$ & $2(2.8)$ & $8(3)$ \\
\hline Student/trainee/fellow & $5(2.6)$ & $4(5.6)$ & $9(3.4)$ \\
\hline Chief Clinical Informatics Officer/Chief Information Officer $(\mathrm{CCIO} / \mathrm{CIO})$ & $2(1)$ & $0(0)$ & $2(0.8)$ \\
\hline Physician assistant & $1(0.5)$ & $1(1.4)$ & $2(0.8)$ \\
\hline Behavioral scientist & $0(0)$ & $1(1.4)$ & $1(0.4)$ \\
\hline Pharmacist & $0(0)$ & $1(1.4)$ & $1(0.4)$ \\
\hline Radiologist & $0(0)$ & $1(1.4)$ & $1(0.4)$ \\
\hline Other & $11(5.7)$ & $2(2.8)$ & $13(4.9)$ \\
\hline Not specified & $0(0)$ & $0(0)$ & $0(0)$ \\
\hline \multicolumn{4}{|l|}{ Setting $^{\mathrm{b}}$} \\
\hline Academia & $63(32.6)$ & $30(42.3)$ & $93(35.2)$ \\
\hline Community-based organization & $10(5.2)$ & $2(2.8)$ & $12(4.5)$ \\
\hline Emergency department & $6(3.1)$ & $0(0)$ & $6(2.3)$ \\
\hline Government & $9(4.7)$ & $2(2.8)$ & $11(4.3)$ \\
\hline Health IT vendor & $14(7.3)$ & $7(9.9)$ & $21(8)$ \\
\hline Health plan & $1(0.5)$ & $1(1.4)$ & $2(0.8)$ \\
\hline Health system & $92(47.7)$ & $24(33.8)$ & $116(43.9)$ \\
\hline Hospital & $66(32.4)$ & $23(32.4)$ & $89(33.7)$ \\
\hline Industry & $8(4.1)$ & $8(11.3)$ & $16(6.1)$ \\
\hline Military & $4(2.1)$ & $1(1.4)$ & 5 (1.9) \\
\hline Nonprofit organization & $18(9.3)$ & 7 (9.9) & $25(9.5)$ \\
\hline Primary care & $25(13)$ & $6(8.5)$ & $31(11.7)$ \\
\hline Private practice & $5(2.6)$ & $0(0)$ & $5(1.9)$ \\
\hline Urgent care/walk-in clinic & $2(1)$ & $0(0)$ & $2(0.8)$ \\
\hline Other & $11(5.7)$ & $5(7)$ & $16(6.1)$ \\
\hline Not specified & $1(0.5)$ & $1(1.4)$ & $2(0.8)$ \\
\hline \multicolumn{4}{|l|}{ Specialty $^{\mathrm{b}}$} \\
\hline Internal medicine & $52(26.9)$ & $13(18.3)$ & $65(24.6)$ \\
\hline Pediatrics & $12(6.2)$ & $9(12.7)$ & $21(8)$ \\
\hline Obstetrics and gynecology & $4(2.1)$ & $5(7)$ & $9(3.4)$ \\
\hline Emergency medicine & $10(5.2)$ & $2(2.8)$ & $12(4.5)$ \\
\hline Psychiatry & $7(3.6)$ & $1(1.4)$ & $8(3)$ \\
\hline
\end{tabular}


Table 1 (Continued)

\begin{tabular}{|c|c|c|c|}
\hline Demographic variable & $\begin{array}{l}\text { Complete } \\
N(\%)\end{array}$ & $\begin{array}{l}\text { Incomplete } \\
N(\%)\end{array}$ & $\begin{array}{l}\text { Totals } \\
N(\%)\end{array}$ \\
\hline Surgery & $3(1.6)$ & $2(2.8)$ & $5(1.9)$ \\
\hline Physical medicine and rehabilitation & $1(0.5)$ & $1(1.4)$ & $2(0.8)$ \\
\hline Radiology & $0(0)$ & $1(1.4)$ & $1(0.4)$ \\
\hline Plastic surgery & $0(0)$ & $1(1.4)$ & $1(0.4)$ \\
\hline Radiation oncology & $0(0)$ & $1(1.4)$ & $1(0.4)$ \\
\hline Family medicine & $13(6.7)$ & $0(0)$ & $13(4.9)$ \\
\hline Anesthesiology & $3(1.6)$ & $0(0)$ & $3(1.1)$ \\
\hline Neurology & $2(1)$ & $0(0)$ & $2(0.8)$ \\
\hline Orthopaedic surgery & $2(1)$ & $0(0)$ & $2(0.8)$ \\
\hline Preventive medicine & $3(1.6)$ & $0(0)$ & $3(1.1)$ \\
\hline Ophthalmology & $1(0.5)$ & $0(0)$ & $1(0.5)$ \\
\hline Other & $46(23.8)$ & $14(19.7)$ & $60(22.7)$ \\
\hline Not applicable & $26(13.5)$ & $18(25.4)$ & $42(16.7)$ \\
\hline Not specified & 23 (11.9) & $8(11.3)$ & $31(11.7)$ \\
\hline \multicolumn{4}{|l|}{ Role categories ${ }^{c}$} \\
\hline Prescribing provider & $88(45.6)$ & $21(29.6)$ & $109(41.3)$ \\
\hline Registered nurse & $66(34.2)$ & $30(42.3)$ & $96(36.4)$ \\
\hline Other & $39(20.2)$ & $20(28.2)$ & $59(22.3)$ \\
\hline
\end{tabular}

${ }^{\text {aRow }}$ percentages.

${ }^{\mathrm{b}}$ Not mutually exclusive categories (participants selected up to three choices).

'Prescribing providers consist of physicians, advance practice nurses, and physician assistants.

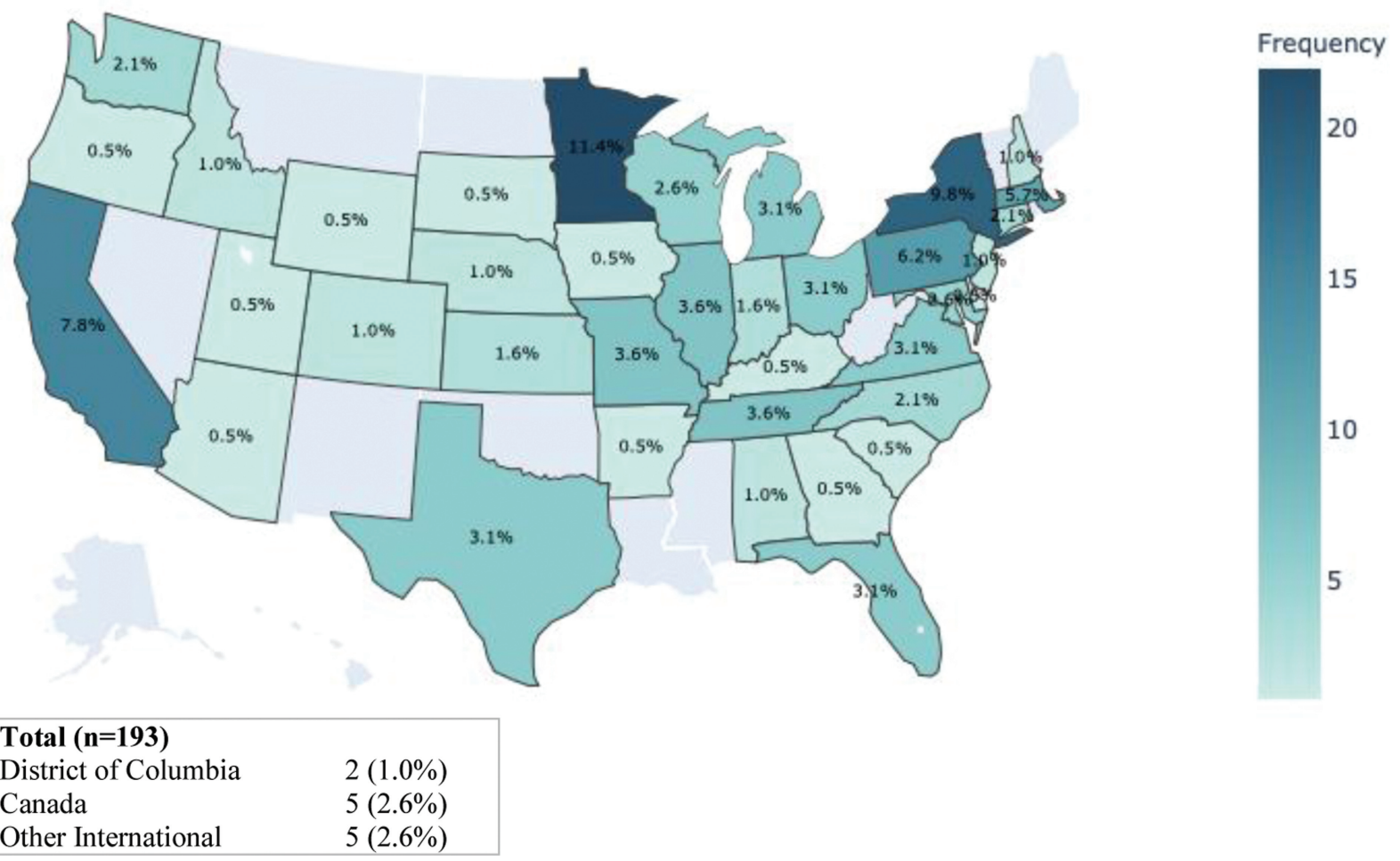

Fig. 1 Location of survey completion by state $(n=193)$. 


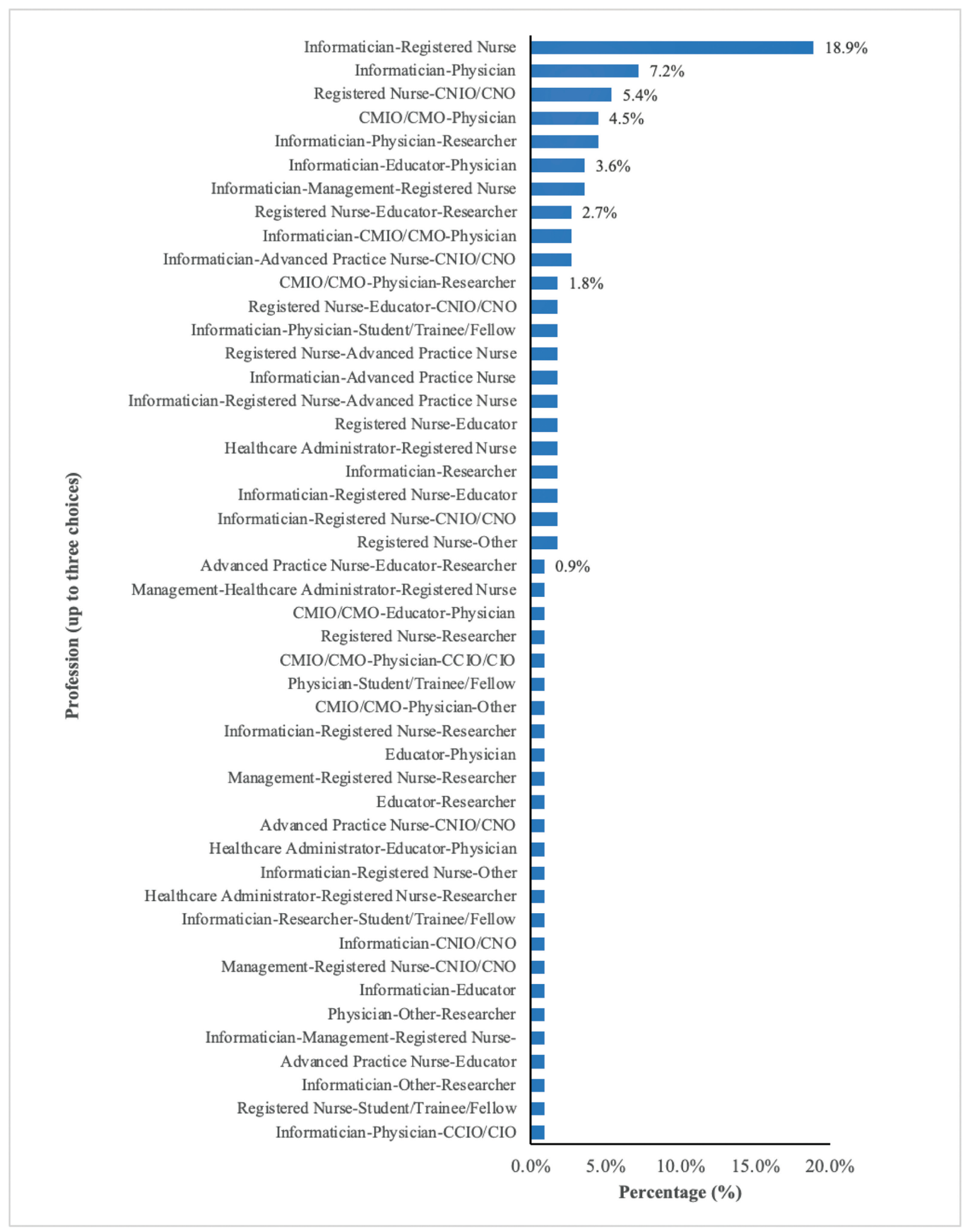

Fig. 2 Distribution of co-occurring roles among respondents who completed the survey $(n=193)$.

\section{Qualitative Analysis}

Additional Experiences Reported in Free-Text Responses We received 59 valid free-text responses regarding additional pandemic-related experiences with clinical documenta- tion reduction. Most responses focused on the following burden domains ${ }^{24}$ : self-imposed $(n=30)$, usability $(n=30)$, quality $(n=20)$, and interoperability/standards $(n=18)$; fewer responses centered around regulatory $(n=10)$ and reimbursement $(n=10)$. Responses were not mutually exclusive 


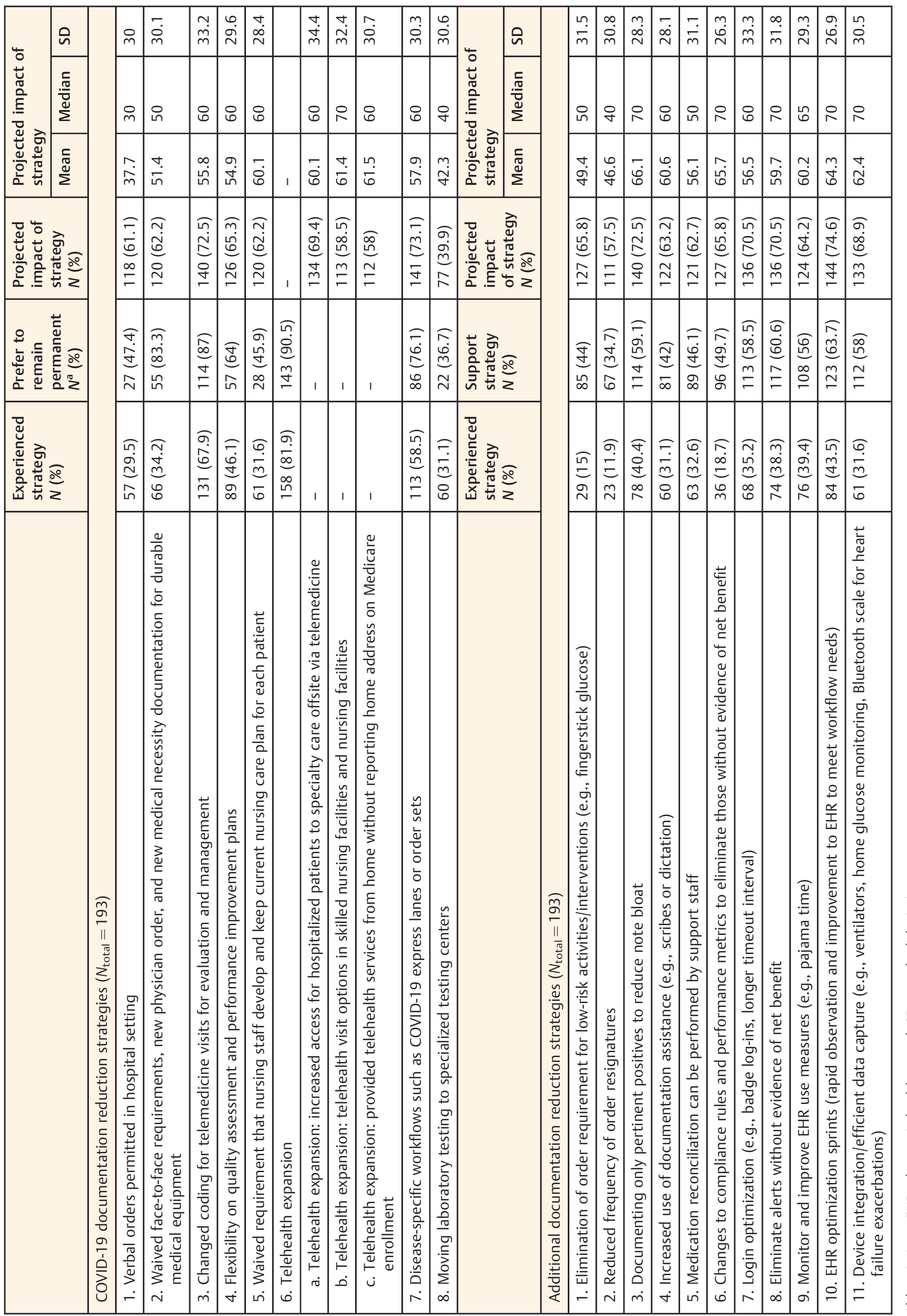

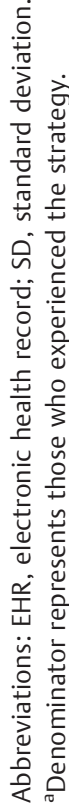



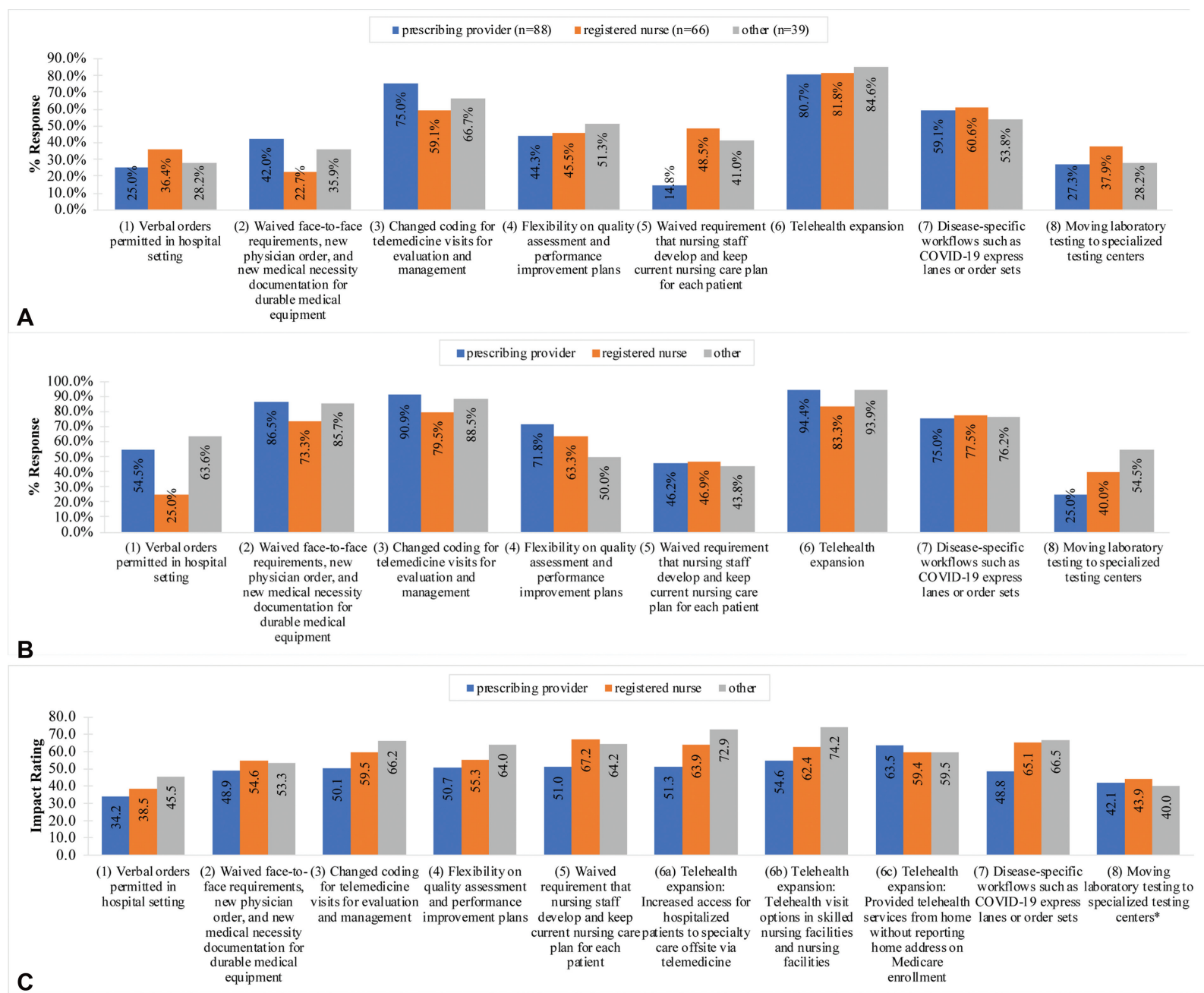

Fig. 3 (A) COVID-19 documentation reduction strategies experienced among completed surveys stratified by role category $(n=193)$ : proportion of respondents that experienced each COVID-19 documentation reduction strategy stratified by role category. (B) COVID-19 documentation reduction strategies experienced among completed surveys stratified by role category $(n=193)$ : among respondents who experienced each COVID-19 documentation reduction strategy, proportion of respondents that preferred COVID-19 documentation reduction strategy to remain permanent stratified by role category. (C) COVID-19 documentation reduction strategies experienced among completed surveys stratified by role category $(n=193)$ : average (rated) projected impact for COVID-19 documentation reduction strategy stratified by role category. ${ }^{*}$ Response rates among at least one role category is $<50 \%$.

and often spanned multiple burden domains. ${ }^{24}$ Eleven invalid responses comprised of survey feedback or reports of no changes.

Themes in the self-imposed domain included using more patient-entered data, re-evaluating system policies (e.g., instituting verbal consent, sprint teams, daily huddles), adding EHR tools to facilitate documentation workflows, and modifying documentation behaviors (see - Supplementary Table S3 [available in the online version only]). Usabilityrelated burden reduction strategies were similarly harmonizing documentation workflows with the EHR, employing artificial intelligence and voice recognition technologies, designing better EHR tools (e.g., templates, visualizations, autogenerated data), integrated devices for documentation, and removing alerts. Quality-related strategies referenced reducing documentation related to screening, care plans, and patient/family education, and charting pertinent positives. Interoperability/standards-based strategies involved integrated devices for documentation, eliminating note redun- dancies and standardizing/modifying documentation procedures, reducing data elements, and charting pertinent positives. Regulatory-specific strategies described adherence to telehealth, CMS, and E\&M code guidelines in addition to billing requirements. Comparably, reimbursement strategies focused on billing requirements, for example, what is billable (e.g., telehealth, time) and whose notes are billable (e.g., medical students).

Eighty-seven participants (45.1\%) reported additional changes to documentation burden at any time (i.e., not immediately linked to COVID-19). Several similar strategies reported were perceived as increasing or decreasing burden by different participants (see - Supplementary Table 54 [available in the online version only]). For example, "bloated templates" and "documentation templates to document on COVID-19 confirmed cases and their discharge disposition" were identified as contributing to burden, while "smart templates to nursing admission history forms to display COVID order[s]" were reported as reducing burden. In 

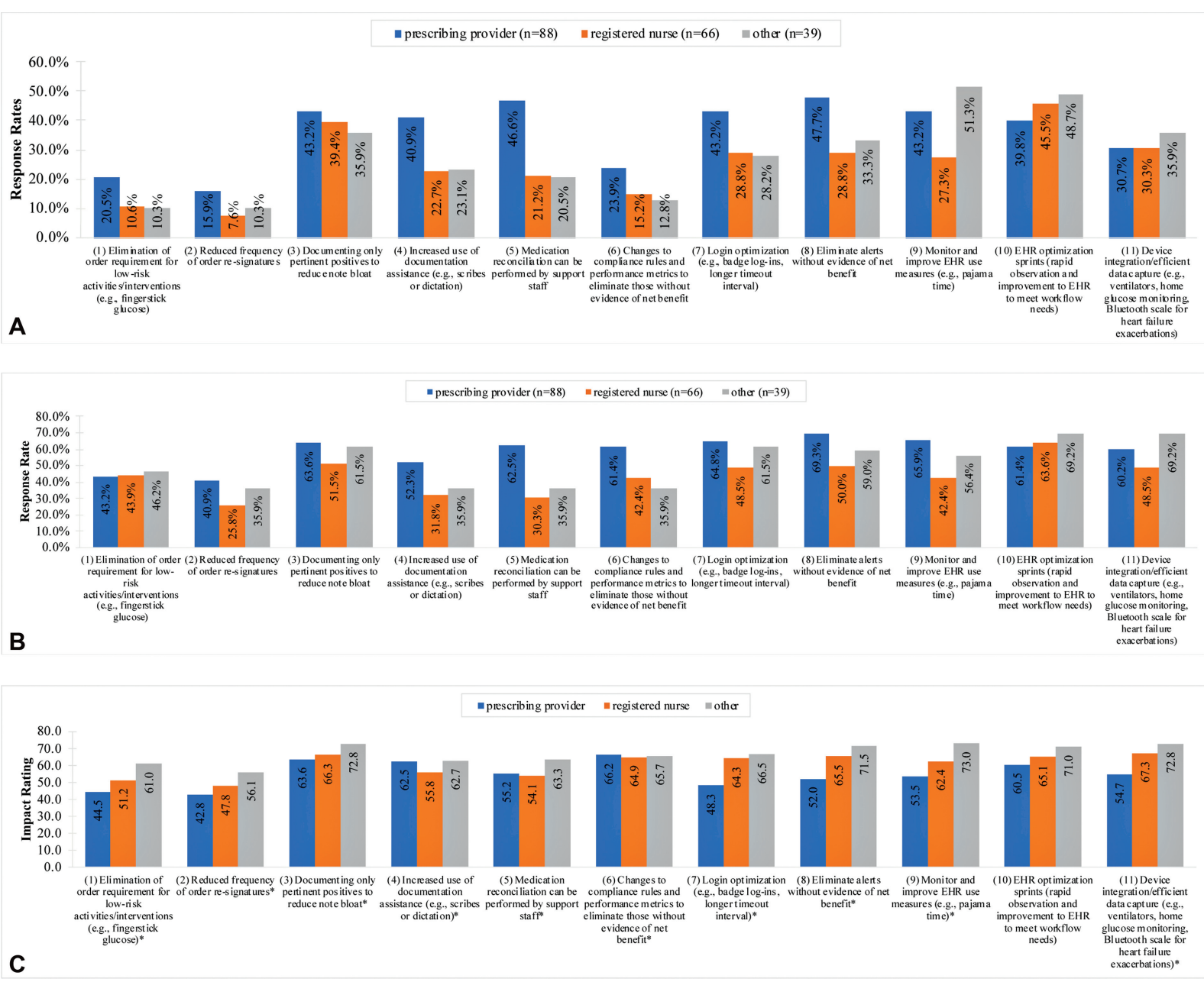

Fig. 4 (A) Additional documentation reduction strategies experienced among completed surveys stratified by role category: proportion of respondents that experienced each COVID-19 documentation reduction strategy stratified by role category. (B) Additional documentation reduction strategies experienced among completed surveys stratified by role category: among respondents who experienced each COVID-19 documentation reduction strategy, proportion of respondents that preferred COVID-19 documentation reduction strategy to remain permanent stratified by role category. (C) Additional documentation reduction strategies experienced among completed surveys stratified by role category: average (rated) projected impact for COVID-19 documentation reduction strategy stratified by role category. *Response rates among at least one role category is $<50 \%$.

reference to "charting pertinent positives," one participant stated the "approach missed a lot of important information that then had to be conveyed in [the] nursing shift report and morning physician report, so in the long run it increased overall burden and decreased ability to care for patients"; another participant wrote, "[r]educe[d] screening by nurses on admission by automating record review and only bringing forward the need to assess if information not in the record" eased burden.

\section{Discussion}

We conducted a nationwide survey among clinicians and health care leaders to assess their experiences and perceptions associated with COVID-19 documentation reduction strategies to understand which strategies should be prioritized and remain permanent post-pandemic. While some strategies were experienced at low proportions, many strategies targeting burden were imposed by health systems. We found that a majority experienced telehealth expansion dur- ing the pandemic and preferred that it remain permanent. Compared with other strategies, telehealth expansion strategies were all rated moderately high impact and over twothirds experienced telehealth coding changes for E\&M. While most participants preferred that these coding changes remain, participants rated these changes as slightly less impactful than individual telehealth initiatives described in the survey. These results are consistent with existing literature as the relaxation of regulations facilitated telehealth uptake and expansion, which, in turn, solved logistical challenges of simultaneously delivering care and maintaining safety during the pandemic. ${ }^{25}$ Given these findings and well-documented inconsistencies in telehealth roll-out across institutions ${ }^{26}$ and states ${ }^{27}$ during the pandemic, additional efforts should be dedicated to developing a long-term regulatory framework (i.e., guidance on infrastructure, reimbursement, licensure) informed by COVID-19 experiences, eliminating barriers to expansion, ${ }^{27,28}$ and developing telehealth platforms that are well integrated into electronic documentation workflows. ${ }^{28}$ 
Less than one-third of participants experienced moving laboratory testing to specialized testing centers or permitting verbal orders in hospital setting, which were also consistent with being least preferred to remain and rated low impact comparatively. However, these strategies may not have been relevant across all participants. Low experience of and preference for strategies were not consistently linked to low impact. For example, waiving requirements on nursing care plans for patients had a moderately high impact rating, particularly among registered nurses.

Overall, participants were more inclined to support additional documentation reduction strategies that directly involved EHR usability (e.g., eliminating alerts, login optimization, EHR optimization sprints, monitoring and improving EHR use measures) and data entry (e.g., documenting only pertinent positives, device integration/efficient data capture) compared with shifting work to auxiliary staff (e.g., documentation assistance, medication reconciliation); nevertheless, contrasts between health care roles were subtle but notable. Prescribing providers were more likely to prefer verbal orders, and support documentation assistance and medication reconciliation performed by support staff compared with other roles, suggesting the electronic documentation ecosystem must be holistically considered when addressing burden to forestall offloading work onto other roles. Documenting only pertinent positives, changing compliance rules and performance metrics to eliminate those without evidence of net benefit, and EHR optimization sprints were rated highest impact compared with other strategies. Among these strategies, implementation was fairly low (range: 40.4-18.7\%). As these strategies address different and interconnecting domains of documentation burden-reimbursement, regulatory, quality, usability, interoperability/standards, and self-imposed ${ }^{24}$-this implies that multifactorial solutions will be required. Despite exhibiting low implementation among participants, several additional strategies were supported by participants at nearly a twofold increase (e.g., eliminating alerts), suggesting the inertia may be associated with organizational culture. The optimal approach to preliminarily prioritize reduction strategies may involve targeting strategies that are highly preferred or supported and rated high impact, and understanding why strategies rated highly impactful were less preferred or supported.

The results of the free-text responses demonstrate that the experience of documentation burden is highly nuanced; perceptions of strategies increasing or decreasing burden pertain to who is reporting it. Templates, adding content to the EHR, and reduced documentation requirements all were described as increasing and decreasing burden. While many expressed "charting by exception" reduced burden, a number stated it missed important information and led to additional work, suggesting considerable variability in the perception and experience of documentation burden exists. These findings indicate that documentation reduction approaches targeting the elimination of documentation irrelevant to the clinical encounter among frontline clinicians must ensure that concision and precision do not come at a cost to the continuity of high-quality, safe patient care.

\section{Limitations}

The survey items were based on Sinsky and Linzer's COVID19 documentation reduction strategies ${ }^{19}$ and those suggested by our experts, which may not be exhaustive or representative of all pandemic-related strategies implemented. ${ }^{19}$ To mitigate this limitation, we included freetext questions to capture any unlisted documentation reduction strategies. Also, we did not evaluate survey reliability. Some strategies were irrelevant to some participants, such as "verbal orders permitted in hospital setting," while "prefer [to] remain permanent" may be ambiguous as it does not clarify the hypothetical situation if the strategy had not already been implemented at their institution; in fact, most questions associated with preference that a strategy remain captured fewer responses relative to experiencing the strategies. As with all self-reported data, responses may be subject to response bias. Our sampling strategy relied on professional listservs and social media, which may not be representative of all clinicians and health care leaders who experienced clinical documentation burden; many participants identified as informaticians and were traced to five states. Due to small sample size, stratified results may not be fully interpretable (-Fig. 3 and -Fig. 4). While this confines the generalizability of our findings, our approach was optimal for achieving a broad understanding of burden under rapidly evolving circumstances of the pandemic. Finally, selection bias is possible depending on whether clinical documentation burden and/or burnout influenced a participants' likelihood of survey completion. Those who identified as informaticians and/or registered nurses had proportionally more partial surveys compared with completed surveys (-Table 1); however, we cannot ascertain if the survey was irrelevant to these participants or if they were interrupted midcompletion.

\section{Future Directions}

We will solicit partnerships with key changemakers to achieve the goal of reducing overall documentation burden by $75 \%$ over the next 5 years, which may result in documentation increases and/or decreases depending on each individual clinical context. These efforts will include reassessing the perceived impact of COVID-19 policies and others implemented to reduce burden, while considering tradeoff between data reduction and data capture. ${ }^{29}$ Concurrent efforts must be dedicated to investigating approaches to gather clinical information without imposing on time clinicians spend engaging in direct patient care, and discovering innovative methods to apply communication and information technology (e.g., artificial intelligence, improved data models) to alleviate documentation-related stress and burnout.

\section{Conclusion}

Natural experiments, such as the COVID-19 pandemic, provide an opportunity to broadly investigate "crisis-related policy and practice changes." ${ }^{19}$ Using Sinsky and Linzer's ${ }^{19}$ recommendations, we developed and distributed an expertvalidated survey to assess pandemic-related documentation 
reduction strategies that clinicians and health care leaders experienced. We found that a large majority experienced telehealth expansion. Compared with other strategies, participants rated telehealth strategies as highest impact on burden reduction. Subtle but notable differences were observed across health care roles. These results will inform the best approaches to decrease documentation burden in the post-COVID era.

\section{Clinical Relevance Statement}

Increased adoption and use of EHRs have catalyzed clinical documentation burden as an issue of a national concern. Documentation burden has intensified clinician burnout and is linked to adverse effects on patient care including increased medical errors and hospital-acquired infections. The $25 \times 5$ Symposium assembled experts from diverse sectors to examine proximal and distal approaches for reducing and, ultimately, eliminating clinical documentation burden. The results of this survey provide insight on documentation reduction strategies implemented during the pandemic, and which strategies clinicians and other health care leaders prefer to remain, are willing to support, and deem high impact. These results will help move the needle toward achieving the Quadruple Aim.

\section{Multiple Choice Questions}

1. Which of the following represents the domains of burden outlined in the American Nursing Informatics Association $(\text { ANIA })^{24}$ conceptual framework to address burden in the EHR?

a. Reimbursement, regulatory, self-imposed, usability.

b. Reimbursement, regulatory, quality, usability, interoperability/standards, self-imposed.

c. Regulatory, quality, documentation, organizational, usability, interoperability/standards.

d. Regulatory, quality, usability, interoperability/standards, self-imposed, reporting.

Correct Answer: The correct answer is option b. The ANIA $^{24}$ framework comprises six domains of burden that intersect at varying degrees.

2. The Quadruple Aim ${ }^{14}$ emerged primarily to address this most recent aim:

a. Reducing costs.

b. Enhancing patient experiences.

c. Improving the work-life balance of the health care provider.

d. Improving population health.

Correct Answer: The correct answer is c. The Quadruple Aim emerged from the Institute for Healthcare Improvement's Triple Aim framework for optimizing health system performance to address the growing threat of clinician burnout on health care outcomes. ${ }^{14}$
3. Which COVID-19 documentation reduction strategy did most respondents experience and prefer to remain permanent?

a. Telehealth expansion.

b. Documenting only pertinent positives.

c. Documentation assistance (e.g., scribes or dictation).

d. Disease-specific workflows.

Correct Answer: The correct answer is a. Over $80 \%$ of survey respondents reported experiencing telehealth expansion during the COVID-19 pandemic. Additionally, over $90 \%$ of survey respondents preferred that telehealth expansion remain permanent.

Author Contributions

A. J. M., S. C. R., and J. M. S. initiated the project, methods, and analysis plan. A. J. M. drafted the manuscript and conducted the quantitative analysis. A. J. M. and J. W. conducted the qualitative analysis. J. M. S. and A. J. M. led the survey development and distribution. J. M. S., E. L., K. D. C., J. W., J. M., S. T. R., and S. C. R. contributed to edits. All members of the $25 \times 5$ Steering Committee contributed as authors, tested the survey instrument, provided conceptual feedback, and approved the final manuscript.

\section{Protection of Human and Animal Subjects}

The study was performed in compliance with the World Medical Association Declaration of Helsinki on Ethical Principles for Medical Research Involving Human Subjects and was reviewed by the Columbia University Irving Medical Center Institutional Review Board.

\section{Funding}

The $25 \times 5$ Symposium was supported through the U.S. National Library of Medicine (NLM) grant 1R13LM013581-01 and co-sponsored by AMIA. The coauthors are also supported through training grants from the NLM 5T15LM007079 and the National Institute for Nursing Research (NINR) 5T32NR007969.

\section{Conflict of Interest}

None declared.

\section{Acknowledgments}

We thank all the informatics experts who provided feedback on our survey and the health care providers and leaders who participated in this project, and Jeff Williamson and Craig Sachson for their support in distributing the survey.

\section{References}

1 HealthIT.gov. Strategy on reducing burden relating to the use of health IT and EHRs [Internet]. Cited June 20, 2020 at: https:// www.healthit.gov/topic/usability-and-provider-burden/strategyreducing-burden-relating-use-health-it-and-ehrs

2 Arndt BG, Beasley JW, Watkinson MD, et al. Tethered to the EHR: primary care physician workload assessment using EHR event log 
data and time-motion observations. Ann Fam Med 2017;15(05): 419-426

3 Sinsky C, Colligan L, Li L, et al. Allocation of physician time in ambulatory practice: a time and motion study in 4 specialties. Ann Intern Med 2016;165(11):753-760

4 Roumeliotis N, Parisien G, Charette S, Arpin E, Brunet F, Jouvet P. Reorganizing care with the implementation of electronic medical records: a time-motion study in the PICU. Pediatr Crit Care Med 2018;19(04):e172-e179

5 Schenk E, Schleyer R, Jones CR, Fincham S, Daratha KB, Monsen KA. Time motion analysis of nursing work in ICU, telemetry and medical-surgical units. J Nurs Manag 2017;25(08):640-646

6 Yen PY, Kellye M, Lopetegui M, et al. Nurses' time allocation and multitasking of nursing activities: a time motion study. AMIA Annu Symp Proc 2018;2018:1137-1146

7 Collins S, Couture B, Kang MJ, et al. Quantifying and visualizing nursing flowsheet documentation burden in acute and critical care. AMIA Annu Symp Proc 2018;2018:348-357

8 Overhage JM, McCallie D Jr. Physician time spent using the electronic health record during outpatient encounters: a descriptive study. Ann Intern Med 2020;172(03):169-174

9 Kroth PJ, Morioka-Douglas N, Veres S, et al. Association of electronic health record design and use factors with clinician stress and burnout. JAMA Netw Open 2019;2(08):e199609

10 Melnick ER, Dyrbye LN, Sinsky CA, et al. The association between perceived electronic health record usability and professional burnout among US physicians. Mayo Clin Proc 2020;95(03): 476-487

11 Topaz M, Ronquillo C, Peltonen LM, et al. Nurse informaticians report low satisfaction and multi-level concerns with electronic health records: results from an international survey. AMIA Annu Symp Proc 2017;2016:2016-2025

12 Gluckman TJ, Vavricek JJ. Streamlining evaluation and management payment to reduce clinician burden. Circ Cardiovasc Qual Outcomes 2019;12(04):e005426

13 Kuhn T, Basch P, Barr M, Yackel TMedical Informatics Committee of the American College of Physicians. Clinical documentation in the 21st century: executive summary of a policy position paper from the American College of Physicians. Ann Intern Med 2015; 162(04):301-303

14 Bodenheimer T, Sinsky C. From Triple to Quadruple Aim: care of the patient requires care of the provider. Ann Fam Med 2014;12 (06):573-576

15 Bachynsky N. Implications for policy: the Triple Aim, Quadruple Aim, and interprofessional collaboration. Nurs Forum 2020;55 (01):54-64

16 Reeves JJ, Hollandsworth HM, Torriani FJ, et al. Rapid response to COVID-19: health informatics support for outbreak management in an academic health system. J Am Med Inform Assoc 2020;27 (06):853-859

17 Grange ES, Neil EJ, Stoffel M, et al. Responding to COVID-19: the UW Medicine Information Technology Services experience. Appl Clin Inform 2020;11(02):265-275

18 HHS.gov. Telehealth: delivering care safely during COVID-19 [Internet]. Available at: https://www.hhs.gov/coronavirus/telehealth/index.html. Cited April 24, 2021

19 Sinsky C, Linzer M. Practice and policy reset post-COVID-19: reversion, transition, or transformation? Health Aff (Millwood) 2020;39(08):1405-1411

20 Cuomo GA. No. 202.10: continuing temporary suspension and modification of laws relating to the disaster emergency. Available at: https://www.governor.ny.gov/news/no-20210-continuingtemporary-suspension-and-modification-laws-relating-disaster-emergency. Cited April 24, 2021

21 Qualtrics. Qualtrics XM // The Leading Experience Management Software. Qualtrics. 2021

22 AMIA. Informatics Professionals. Leading the Way [Internet]. Available at: https://www.amia.org/. Cited April 24, 2021

23 Polit DF, Beck CT. The content validity index: are you sure you know what's being reported? Critique and recommendations. Res Nurs Health 2006;29(05):489-497

24 Sengstack PP, Adrian B, David R-B, Boyd L, Davis A, Hook M, et al. The six domains of burden: a conceptual framework to address the burden of documentation in the electronic health record. Position Paper of the American Nursing Informatics Association Board of Directors. Available at: https://www.ache.org/-/media/ ache/about-ache/corporate-partners/the-six-domains-of-burden_cerner-documentation.pdf. Cited May 1, 2021

25 Temesgen ZM, DeSimone DC, Mahmood M, Libertin CR, Varatharaj Palraj BR, Berbari EF. Health care after the COVID-19 pandemic and the influence of telemedicine. Mayo Clin Proc 2020;95(9S):S66-S68

26 Hincapié MA, Gallego JC, Gempeler A, Piñeros JA, Nasner D, Escobar MF. Implementation and usefulness of telemedicine during the COVID-19 pandemic: a scoping review. J Prim Care Community Health 2020;11:2150132720980612

27 Lee NT, Karsten J, Roberts J. Removing regulatory barriers to telehealth before and after COVID-10. Accessed August 22, 2021 at: https://www.brookings.edu/research/removing-regulatory-barriers-to-telehealth-before-and-after-covid-19/. Published May 2020

28 Lieneck C, Garvey J, Collins C, Graham D, Loving C, Pearson R. Rapid telehealth implementation during the COVID-19 global pandemic: a rapid review. Healthcare (Basel) 2020;8(04):517

29 Johnson KB, Neuss MJ, Detmer DE. Electronic health records and clinician burnout: a story of three eras. J Am Med Inform Assoc 2021;28(05):967-973 